$9-17-2020$

\title{
Concomitant of Order Statistics from New Bivariate Gompertz Distribution
}

Sumit Kumar

Babasaheb Bhimrao Ambedkar University, sumitkumar130586@gmail.com

M. J. S. Khan

Aligarh Muslim University, jahangirskhan@gmail.com

Surinder Kumar

Babashaheb Bhimrao Ambedkar University, surinderntls@gmail.com

Follow this and additional works at: https://digitalcommons.wayne.edu/jmasm

Part of the Applied Statistics Commons, Social and Behavioral Sciences Commons, and the Statistical Theory Commons

\section{Recommended Citation}

Kumar, S., Khan, M. J. S., \& Kumar, S. (2019). Concomitant of order statistics from new bivariate Gompertz distribution. Journal of Modern Applied Statistical Methods, 18(2), eP2826. doi: 10.22237/jmasm/ 1604189820

This Regular Article is brought to you for free and open access by the Open Access Journals at DigitalCommons@WayneState. It has been accepted for inclusion in Journal of Modern Applied Statistical Methods by an authorized editor of DigitalCommons@WayneState. 


\title{
Concomitant of Order Statistics from New Bivariate Gompertz Distribution
}

\author{
Sumit Kumar \\ Babasaheb Bhimrao Ambedkar University \\ Lucknow, India
}

\author{
M. J. S. Khan \\ Aligarh Muslim University \\ Aligarh, India
}

\author{
Surinder Kumar \\ Babasaheb Bhimrao Ambedkar University \\ Lucknow, India
}

For the new bivariate Gompertz distribution, the expression for probability density function (pdf) of $r^{\text {th }}$ order statistics and pdf of concomitant arising from $r^{\text {th }}$ order statistics are derived. The properties of concomitant arising from the corresponding order statistics are used to derive these results. The exact expression for moment generating function (mgf) of concomitant of order $r^{\text {th }}$ statistics is derived. Also, the mean of concomitant arising from $r^{\text {th }}$ order statistics is computed using the mgf of concomitant of $r^{\text {th }}$ order statistics, and the exact expression for joint density of concomitant of two non-adjacent order statistics are derived.

Keywords: $\quad$ New bivariate Gompertz distribution, order statistics, concomitant of order statistics, moment generating function

\section{Introduction}

In the theory of concomitant of order statistics by David (1973), the values assumed by concomitant variable $Y$ are corresponding to each value of variable $X$, arranged in some specified order, from a bivariate population $\left(X_{i}, Y_{i}\right) ; i=1,2, \ldots, n$. Concomitant of order statistics finds frequent application in selection problems, where decision of selection is based on the values of two random variables $X$ and $Y$. For, e.g., $X$ may be the score of students in preliminary examination and $Y$ may be the corresponding score in final examination, $X$ may be the height of an individual and $Y$ may be the body weight of same individual in a certain physical examination test, etc.

doi: 10.22237/jmasm/1604189820 | Accepted: June 26, 2018; Published: September 17, 2020.

Correspondence: M. J. S. Khan, jahangirskhan@gmail.com 


\section{KUMAR ET AL}

El-Sherpieny et al. (2013) discussed a new bivariate Gompertz distribution, denoted by $\operatorname{NBG}\left(\alpha_{1}, \alpha_{2}, \alpha_{3}, \lambda\right)$ which has Gompertz marginals. Univariate Gompertz distribution with parameters $\alpha, \lambda$ has the probability density function (pdf) is as follows:

$$
\mathrm{f}(x, \alpha, \lambda)=\alpha \mathrm{e}^{\lambda x} \mathrm{e}^{-\frac{\alpha}{\lambda}\left(\mathrm{e}^{\lambda x}-1\right)} ; \quad \alpha, \lambda, x>0
$$

and the cumulative distribution function (cdf) of univariate Gompertz distribution is given by

$$
\mathrm{F}(x, \alpha, \lambda)=1-\mathrm{e}^{-\frac{\alpha}{\lambda}\left(\mathrm{e}^{\lambda x}-1\right)}
$$

Suppose $U_{i} \sim \mathrm{G}\left(\alpha_{i}, \lambda\right), i=1,2,3$, are independently distributed. If we consider the random variable $X=\min \left(U_{1}, U_{2}\right)$ and $Y=\min \left(U_{2}, U_{3}\right)$, then the random vector $(X, Y)$ shall follow $\operatorname{NBG}\left(\alpha_{1}, \alpha_{2}, \alpha_{3}, \lambda\right), \alpha_{1}, \alpha_{2}, \alpha_{3}, \lambda>0$, with corresponding joint cdf can be written as

$$
\mathrm{F}(x, y)= \begin{cases}\mathrm{F}_{1}(x, y) ; & \text { if } 0<y<x \\ \mathrm{~F}_{2}(x, y) ; & \text { if } 0<x<y \\ \mathrm{~F}_{3}(x, y) ; & \text { if } y=x>0\end{cases}
$$

where

$$
\begin{aligned}
& \mathrm{F}_{1}(x, y)=1-\left[\mathrm{e}^{-\left(\frac{\alpha_{1}+\alpha_{3}}{\lambda}\right)\left(\mathrm{e}^{\lambda x}-1\right)} \mathrm{e}^{-\frac{\alpha_{2}}{\lambda}\left(\mathrm{e}^{\lambda y}-1\right)}\right] \\
& \mathrm{F}_{2}(x, y)=1-\left[\mathrm{e}^{-\frac{\alpha_{1}}{\lambda}\left(\mathrm{e}^{\lambda x}-1\right)} \mathrm{e}^{-\left(\frac{\alpha_{2}+\alpha_{3}}{\lambda}\right)\left(\mathrm{e}^{\lambda y}-1\right)}\right] \\
& \mathrm{F}_{3}(x, y)=1-\left[\mathrm{e}^{-\left(\frac{\alpha_{1}+\alpha_{2}+\alpha_{3}}{\lambda}\right)\left(\mathrm{e}^{\lambda x}-1\right)}\right]
\end{aligned}
$$

and the corresponding joint pdf can be obtained as 


\section{CONCOMITANT OF ORDER STATISTICS}

$$
\mathrm{f}(x, y)= \begin{cases}\mathrm{f}_{1}(x, y) ; & \text { if } 0<y<x \\ \mathrm{f}_{2}(x, y) ; & \text { if } 0<x<y \\ \mathrm{f}_{3}(x, y) ; & \text { if } y=x>0\end{cases}
$$

where

$$
\begin{aligned}
& \mathrm{f}_{1}(x, y)=\alpha_{2}\left(a_{1}+\alpha_{3}\right) \mathrm{e}^{\lambda x} \mathrm{e}^{\lambda y}\left[\mathrm{e}^{-\left(\frac{\alpha_{1}+\alpha_{3}}{\lambda}\right)\left(\mathrm{e}^{\lambda x}-1\right)} \mathrm{e}^{-\frac{\alpha_{2}}{\lambda}\left(\mathrm{e}^{\lambda y}-1\right)}\right] \\
& \mathrm{f}_{2}(x, y)=\alpha_{1}\left(a_{2}+\alpha_{3}\right) \mathrm{e}^{\lambda x} \mathrm{e}^{\lambda y}\left[\mathrm{e}^{-\frac{\alpha_{1}}{\lambda}\left(\mathrm{e}^{\lambda x}-1\right)} \mathrm{e}^{-\left(\frac{\alpha_{2}+\alpha_{3}}{\lambda}\right)\left(\mathrm{e}^{\lambda y}-1\right)}\right] \\
& \mathrm{f}_{3}(x, y)=\alpha_{3} \mathrm{e}^{\lambda x}\left[\mathrm{e}^{-\left(\frac{\alpha_{1}+\alpha_{2}+\alpha_{3}}{\lambda}\right)\left(\mathrm{e}^{\lambda x}-1\right)}\right]
\end{aligned}
$$

Therefore, the marginal pdf and marginal cdf of $X$ can be represented, respectively, as

$$
\begin{gathered}
\mathrm{f}(x)=\left(\alpha_{1}+\alpha_{3}\right) \mathrm{e}^{\lambda x}\left[\mathrm{e}^{-\left(\frac{\alpha_{1}+\alpha_{3}}{\lambda}\right)\left(\mathrm{e}^{\lambda x-1}\right)}\right] ; \quad x>0, \\
\mathrm{~F}(x)=1-\left[\mathrm{e}^{-\left(\frac{\alpha_{1}+\alpha_{3}}{\lambda}\right)\left(\mathrm{e}^{\lambda x}-1\right)}\right] .
\end{gathered}
$$

Thus, the conditional pdf of $Y$ given $X$ is as follows:

$$
\mathrm{f}(y \mid x)= \begin{cases}\mathrm{f}_{1}(y \mid x) ; & \text { if } 0<y<x \\ \mathrm{f}_{2}(y \mid x) ; & \text { if } 0<x<y \\ \mathrm{f}_{3}(y \mid x) ; & \text { if } y=x>0\end{cases}
$$

where

$$
\mathrm{f}_{1}(y \mid x)=\alpha_{2} \mathrm{e}^{\frac{\alpha_{2}}{\lambda}} \mathrm{e}^{\lambda y} \mathrm{e}^{-\frac{\alpha_{2}}{\lambda} \mathrm{e}^{\lambda y}}
$$




$$
\begin{aligned}
& \mathrm{f}_{2}(y \mid x)=\alpha_{1}\left(\frac{\alpha_{2}+\alpha_{3}}{\alpha_{1}+\alpha_{3}}\right) \mathrm{e}^{\lambda y} \mathrm{e}^{-\left(\frac{\alpha_{2}+\alpha_{3}}{\lambda}\right) \mathrm{e}^{\lambda y}} \mathrm{e}^{\frac{\alpha_{2}}{\lambda}} \mathrm{e}^{-\frac{\alpha_{3}}{\lambda} \mathrm{e}^{\lambda x}} \\
& \mathrm{f}_{3}(y \mid x)=\left(\frac{\alpha_{3}}{\alpha_{1}+\alpha_{3}}\right) \mathrm{e}^{-\frac{\alpha_{3}}{\lambda}\left(\mathrm{e}^{\lambda x}-1\right)}
\end{aligned}
$$

Subsequently, Yang (1977) discussed the general distribution theory of the concomitants of order statistics. Bhattacharya (1984) also explained the theory and application of concomitant of order statistics in the name of induced order statistics. Balasubramanian and Beg $(1996,1997)$ obtained expressions of concomitant of order statistics in bivariate exponential distribution of Marshall and Olkin and concomitant of order statistics in Morgenstern type bivariate exponential distribution. Balasubramanian and Beg (1998) studied the concomitant of order statistics arising from Gumbel's bivariate exponential distribution. Further, Begum and Khan (2000) and Begum (2003) obtained expressions of concomitant of order statistics from Marshall and Olkin's bivariate Weibull distribution and bivariate Pareto II distribution. In the same sequence of advancements to the theory of concomitant of order statistics, Thomas and Veena (2011) defined the application of concomitant of order statistics in characterizing a family of bivariate distributions. Chacko and Thomas (2011) and Philip and Thomas (2015) extended the application of concomitant of order statistics in the estimation of parameters of Morgenstern type bivariate exponential distribution and extended Farlie-GumbelMorgenstern bivariate logistic distribution, respectively. In this study, the properties of concomitant of $r^{\text {th }}$ order statistics, i.e. $Y_{[r: n]}$, are studied if the random variables $\left(X_{i}, Y_{i}\right), i=1,2, \ldots, n$, are iid and follows $\operatorname{NBG}\left(\alpha_{1}, \alpha_{2}, \alpha_{3}, \lambda\right)$.

\section{Distribution of Order Statistics}

Let $X_{1}, X_{2}, \ldots, X_{n}$ be the $n$ iid random variables from the population having pdf $\mathrm{f}(x)$ and cdf $\mathrm{F}(x)$. Then the pdf of $r^{\text {th }}$ order statistics $X_{r: n}$ is given by (David, 1981):

$$
\mathrm{f}_{r: n}(x)=C_{r: n}[\mathrm{~F}(x)]^{r-1}[1-\mathrm{F}(x)]^{n-r} \mathrm{f}(x) ; \quad-\infty<x<\infty,
$$

where $C_{r: n}=n ! /(r-1) !(n-r) !$.

Therefore, the pdf of $r^{\text {th }}$ order statistics $X_{r: n}$, when $X_{1}, X_{2}, \ldots, X_{n}$ are iid random variables having pdf and cdf given in (5) and (6), respectively, is given by 


\section{CONCOMITANT OF ORDER STATISTICS}

$$
\begin{aligned}
\mathrm{f}_{r: n}(x)=C_{r: n}\left(\alpha_{1}+\alpha_{3}\right)\left(\mathrm{e}^{\lambda x} \mathrm{e}^{-(n-r+1)\left(\frac{\alpha_{1}+\alpha_{3}}{\lambda}\right) \mathrm{e}^{\lambda x}} \mathrm{e}^{(n-r+1)\left(\frac{\alpha_{1}+\alpha_{3}}{\lambda}\right)}\right. \\
\times\left[1-\mathrm{e}^{\left(\frac{\alpha_{1}+\alpha_{3}}{\lambda}\right) \mathrm{e}^{\lambda x}} \mathrm{e}^{\left.\left.\left(\frac{\alpha_{1}+\alpha_{3}}{\lambda}\right)\right]^{r-1}\right) ; 0<x<\infty}\right.
\end{aligned}
$$

Putting $r=1$ in (8), we get the pdf of the first order statistics $X_{1: n}$ as

$$
\mathrm{f}_{1: n}(x)=n\left(\alpha_{1}+\alpha_{3}\right) \mathrm{e}^{\lambda x} \mathrm{e}^{-n\left(\frac{\alpha_{1}+\alpha_{3}}{\lambda}\right) \mathrm{e}^{\lambda x}} \mathrm{e}^{n\left(\frac{\alpha_{1}+\alpha_{3}}{\lambda}\right)} ; \quad 0<x<\infty .
$$

Let $X_{r: n}$ and $X_{s: n}$ be the two order statistics $(1 \leq r<s \leq n)$; then the joint pdf of two order statistics is given by (David, 1981):

$$
\mathrm{f}_{r, s: n}\left(x_{1}, x_{2}\right)=C_{r, s: n}\left[1-\overline{\mathrm{F}}\left(x_{1}\right)\right]^{r-1}\left[\overline{\mathrm{F}}\left(x_{1}\right)-\overline{\mathrm{F}}\left(x_{2}\right)\right]^{s-r-1}\left[\overline{\mathrm{F}}\left(x_{2}\right)\right]^{n-s} \mathrm{f}\left(x_{1}\right) \mathrm{f}\left(x_{2}\right),
$$

$-\infty<x_{1}<x_{2}<\infty$, where

$$
C_{r, s: n}=\frac{n !}{(r-1) ![s-(r+1)] !(n-s) !}
$$

and $\overline{\mathrm{F}}(x)=[1-\mathrm{F}(x)]$.

Therefore, if $X_{1}, X_{2}, \ldots, X_{n}$ are $n$ iid random variables having pdf and cdf given in (5) and (6), respectively, then the joint pdf of $X_{r: n}$ and $X_{s: n}$ is given by

$$
\begin{aligned}
& \mathrm{f}_{r, s: n}\left(x_{1}, x_{2}\right)= \\
& C_{r, s: n}\left(\alpha_{1}+\alpha_{3}\right)^{2} \mathrm{e}^{\lambda x_{1}} \mathrm{e}^{\lambda x_{2}} \sum_{l=0}^{r-1} \sum_{k=0}^{s-r-1}(-1)^{l+k}\left(\begin{array}{c}
r-1 \\
l
\end{array}\right)\left(\begin{array}{c}
s-r-1 \\
k
\end{array}\right) \\
& \quad \times \mathrm{e}^{-\frac{\left(\alpha_{1}+\alpha_{3}\right)}{\lambda}\left[(l+s-r-k) \mathrm{e}^{\lambda x_{1}}+(n-s+k+1) \mathrm{e}^{\lambda x_{2}}-(l+n-r+1)\right]}
\end{aligned}
$$

\section{Distribution of Concomitant of Order Statistics}

The pdf of concomitant of first order statistics $Y_{[1: n]}$ is given by (Begum \& Khan, 2000) 


\section{KUMAR ET AL}

$$
\mathrm{g}_{[1: n]}(y)=\int_{y}^{\infty} \mathrm{f}_{1}(y \mid x) \mathrm{f}_{1: n}(x) d x+\int_{0}^{y} \mathrm{f}_{2}(y \mid x) \mathrm{f}_{1: n}(x) d x+\mathrm{f}_{3}(y \mid x) \mathrm{f}_{1: n}(x) .
$$

Consider (7) and (9); the pdf of concomitant of first order statistics $Y_{[1: n]}$ is given by

$$
\begin{aligned}
\mathrm{g}_{[1: n]}(y)= & n \alpha_{2}\left(\alpha_{1}+\alpha_{3}\right) \mathrm{e}^{\frac{\alpha_{2}}{\lambda}} \mathrm{e}^{\frac{n\left(\alpha_{1}+\alpha_{3}\right)}{\lambda}} \mathrm{e}^{\lambda y} \mathrm{e}^{-\frac{\alpha_{2}}{\lambda} e^{\lambda y}} \int_{y}^{\infty} \mathrm{e}^{\lambda x} \mathrm{e}^{-\frac{n\left(\alpha_{1}+\alpha_{3}\right)}{\lambda} \mathrm{e}^{\lambda x}} d x \\
& +n \alpha_{1}\left(\alpha_{2}+\alpha_{3}\right) \mathrm{e}^{\lambda y} \mathrm{e}^{-\frac{\left(\alpha_{2}+\alpha_{3}\right)}{\lambda} \mathrm{e}^{\lambda y}} \mathrm{e}^{\frac{\alpha_{2}}{\lambda}} \mathrm{e}^{\frac{n\left(\alpha_{1}+\alpha_{3}\right)}{\lambda}} \int_{0}^{y} \mathrm{e}^{\frac{\alpha_{3}}{\lambda} \mathrm{e}^{\lambda x}} \mathrm{e}^{\lambda x} \mathrm{e}^{-\frac{n\left(\alpha_{1}+\alpha_{3}\right)}{\lambda} \mathrm{e}^{2 x}} d x \\
& +n \alpha_{3} \mathrm{e}^{\frac{n\left(\alpha_{1}+\alpha_{3}\right)}{\lambda}} \mathrm{e}^{-\frac{\alpha_{3}}{\lambda}\left(\mathrm{e}^{\lambda y}-1\right)} \mathrm{e}^{\lambda y} \mathrm{e}^{-\frac{n\left(\alpha_{1}+\alpha_{3}\right)}{\lambda} \mathrm{e}^{\lambda y}}
\end{aligned}
$$

After some algebraic simplification, we get

$$
\begin{aligned}
& \mathrm{g}_{[1: n]}(y)=\alpha_{2} \mathrm{e}^{\lambda y} \mathrm{e}^{\left[\frac{n\left(\alpha_{1}+\alpha_{3}\right)+\alpha_{2}}{\lambda}\right]\left(1-\mathrm{e}^{\lambda y}\right)}+\frac{n \alpha_{1}\left(\alpha_{2}+\alpha_{3}\right)}{\left[n\left(\alpha_{1}+\alpha_{3}\right)-\alpha_{3}\right]} \mathrm{e}^{\lambda y} \mathrm{e}^{\frac{\left(\alpha_{2}+\alpha_{3}\right)}{\lambda}\left(1-\mathrm{e}^{\lambda y}\right)} \\
& -\frac{n \alpha_{1}\left(\alpha_{2}+\alpha_{3}\right)}{\left[n\left(\alpha_{1}+\alpha_{3}\right)-\alpha_{3}\right]} \mathrm{e}^{\lambda y} \mathrm{e}^{\left[\frac{n\left(\alpha_{1}+\alpha_{3}\right)-\alpha_{2}}{\lambda}\right]\left(1-\mathrm{e}^{\lambda y}\right)}+n \alpha_{3} \mathrm{e}^{\lambda y} \mathrm{e}^{\left[\frac{n\left(\alpha_{1}+\alpha_{3}\right)+\alpha_{3}}{\lambda}\right]\left(1-\mathrm{e}^{\lambda y}\right)}
\end{aligned}
$$

The relation between the pdf of concomitant of first order statistics and concomitant of $r^{\text {th }}$ order statistics is given by (Balasubramanian \& Beg, 1998)

$$
\mathrm{g}_{[r: n]}(y)=\sum_{i=n-r+1}^{n}(-1)^{i-n+r-1}\left(\begin{array}{c}
i-1 \\
n-r
\end{array}\right)\left(\begin{array}{l}
n \\
i
\end{array}\right) \mathrm{g}_{[1: i]}(y) \text {. }
$$

Therefore, the pdf of concomitant of $r^{\text {th }}$ order statistics is given, for $0<y<\infty$, by 


\section{CONCOMITANT OF ORDER STATISTICS}

$$
\begin{aligned}
\mathrm{g}_{[r: n]}(y)= & \sum_{i=n-r+1}^{n}(-1)^{i-n+r-1}\left(\begin{array}{c}
i-1 \\
n-r
\end{array}\right)\left(\begin{array}{c}
n \\
i
\end{array}\right)\left[\alpha_{2} \mathrm{e}^{\lambda y} \mathrm{e}^{\left[\frac{i\left(\alpha_{1}+\alpha_{3}\right)+\alpha_{2}}{\lambda}\right]\left(1-\mathrm{e}^{\lambda y}\right)}\right. \\
& +\frac{i \alpha_{1}\left(\alpha_{2}+\alpha_{3}\right)}{\left[i\left(\alpha_{1}+\alpha_{3}\right)-\alpha_{3}\right]} \mathrm{e}^{\lambda y} \mathrm{e}^{\frac{\left(\alpha_{2}+\alpha_{3}\right)}{\lambda}\left(1-\mathrm{e}^{\lambda y}\right)} \\
& \left.-\frac{i \alpha_{1}\left(\alpha_{2}+\alpha_{3}\right)}{\left[i\left(\alpha_{1}+\alpha_{3}\right)-\alpha_{3}\right]} \mathrm{e}^{\lambda y} \mathrm{e}^{\left[\frac{i\left(\alpha_{1}+\alpha_{3}\right)-\alpha_{2}}{\lambda}\right]\left(1-\mathrm{e}^{\lambda y}\right)}+i \alpha_{3} \mathrm{e}^{\lambda y} \mathrm{e}^{\left[\frac{i\left(\alpha_{1}+\alpha_{3}\right)+\alpha_{3}}{\lambda}\right]\left(1-\mathrm{e}^{\lambda y}\right)}\right]
\end{aligned}
$$

\section{Moment Generating Function of Concomitant of Order Statistics}

The moment generating function of concomitant of first order statistics $Y_{[1: n]}$ is defined as

$$
\mathrm{M}_{Y_{[n: n]}}(t)=\int_{0}^{\infty} \mathrm{e}^{t y} \mathrm{~g}_{[1: n]}(y) d y .
$$

Thus, in view of (11), we have:

$$
\begin{aligned}
& \mathrm{M}_{Y_{[[n]}}(t)=\alpha_{2} \mathrm{e}^{\left[\frac{n\left(\alpha_{1}+\alpha_{3}\right)+\alpha_{2}}{\lambda}\right]} \int_{0}^{\infty} \mathrm{e}^{(t+\lambda) y} \mathrm{e}^{\left[\frac{n\left(\alpha_{1}+\alpha_{3}\right)+\alpha_{2}}{\lambda}\right] \mathrm{e}^{\lambda y}} d y \\
& +\frac{n \alpha_{1}\left(\alpha_{2}+\alpha_{3}\right)}{\left[n\left(\alpha_{1}+\alpha_{3}\right)-\alpha_{3}\right]} \mathrm{e}^{\frac{\left(\alpha_{2}+\alpha_{3}\right)}{\lambda}} \int_{0}^{\infty} \mathrm{e}^{(t+\lambda) y} \mathrm{e}^{-\left(\frac{\alpha_{2}+\alpha_{3}}{\lambda} \mathrm{e}^{\mathrm{e}^{y}}\right.} d y \\
& -\frac{n \alpha_{1}\left(\alpha_{2}+\alpha_{3}\right)}{\left[n\left(\alpha_{1}+\alpha_{3}\right)-\alpha_{3}\right]} \mathrm{e}^{\left[\frac{n\left(\alpha_{1}+\alpha_{3}\right)-\alpha_{2}}{\lambda}\right]} \int_{0}^{\infty} \mathrm{e}^{(t+\lambda) y} \mathrm{e}^{\left[\frac{n\left(\alpha_{1}+\alpha_{3}\right)-\alpha_{2}}{\lambda}\right] \mathrm{e}^{\lambda y}} d y \\
& +n \alpha_{3} \mathrm{e}^{\left[\frac{n\left(\alpha_{1}+\alpha_{3}\right)+\alpha_{2}}{\lambda}\right]} \int_{0}^{\infty} \mathrm{e}^{(t+\lambda) y} \mathrm{e}^{-\left[\frac{n\left(\alpha_{1}+\alpha_{3}\right)-\alpha_{3}}{\lambda}\right] \mathrm{e}^{\lambda y}} d y
\end{aligned}
$$

Consider the integral

$$
I_{1}=\int_{0}^{\infty} \mathrm{e}^{(t+\lambda) y} \mathrm{e}^{-\left\{\frac{n\left(\alpha_{1}+\alpha_{3}\right)+\alpha_{2}}{\lambda}\right\} \mathrm{e}^{\lambda y}} d y
$$


and

$$
\theta_{1}=\left\{\frac{n\left(\alpha_{1}+\alpha_{3}\right)+\alpha_{2}}{\lambda}\right\}
$$

after simplification, we get

$$
I_{1}=\frac{1}{\lambda \theta_{1}^{\left(\frac{t}{\lambda}+1\right)}}\left\{\Gamma\left(\frac{t}{\lambda}+1\right)-\gamma\left(\frac{t}{\lambda}+1, \theta_{1}\right)\right\},
$$

where $\Gamma($.$) is the gamma function and \gamma(.,$.$) is the lower incomplete gamma function$ defined as

$$
\gamma(a, x)=\int_{0}^{x} u^{a-1} \mathrm{e}^{-u} d u
$$

Thus, using the relationship between lower incomplete gamma function and Kummer's confluent hypergeometric function, ${ }_{1} \mathrm{~F}_{1}(1, s+1, z)$, which is given by $\gamma(s, z)=s^{-1} z^{s} \mathrm{e}^{-z}{ }_{1} \mathrm{~F}_{1}(1, s+1, z)$, (see Abramowitz and Stegun, 1972, p. 262),

$$
\gamma\left(\frac{t}{\lambda}+1, \theta_{1}\right)=\left(\frac{t}{\lambda}+1\right)^{-1} \theta_{1}^{\left(\frac{t}{\lambda}+1\right)} \mathrm{e}^{-\theta_{1}} \mathrm{~F}_{1}\left(1, \frac{t}{\lambda}+2, \theta_{1}\right) .
$$

This implies

$$
I_{1}=\frac{\Gamma\left(\frac{t}{\lambda}+1\right)}{\lambda \theta_{1}^{\left(\frac{t}{\lambda}+1\right)}}-\frac{\left(\frac{t}{\lambda}+1\right)^{-1} \mathrm{e}^{-\theta_{1}}{ }_{1} \mathrm{~F}_{1}\left(1, \frac{t}{\lambda}+2, \theta_{1}\right)}{\lambda} .
$$

Similarly, denoting

$$
\theta_{2}=\frac{\alpha_{2}+\alpha_{3}}{\lambda}, \theta_{3}=\frac{n\left(\alpha_{1}+\alpha_{3}\right)-\alpha_{2}}{\lambda}, \text { and } \theta_{4}=\frac{n\left(\alpha_{1}+\alpha_{3}\right)+\alpha_{3}}{\lambda},
$$




\section{CONCOMITANT OF ORDER STATISTICS}

respectively, and solving the rest of the integrals of (13), we get the mgf of concomitant of first order statistics given as

$$
\begin{aligned}
& \mathbf{M}_{Y_{[1 \cdot n]}}(t)=\alpha_{2} \mathrm{e}^{\theta_{1}}\left[\frac{\Gamma\left(\frac{t}{\lambda}+1\right)}{\lambda \theta_{1}^{\left(\frac{t}{\lambda}+1\right)}}-\frac{\left(\frac{t}{\lambda}+1\right)^{-1} \mathrm{e}^{-\theta_{1}}{ }_{1} \mathrm{~F}_{1}\left(1, \frac{t}{\lambda}+2, \theta_{1}\right)}{\lambda}\right] \\
& +\frac{n \alpha_{1}\left(\alpha_{2}+\alpha_{3}\right) \mathrm{e}^{\theta_{2}}}{\left[n\left(\alpha_{1}+\alpha_{3}\right)-\alpha_{3}\right]}\left[\frac{\Gamma\left(\frac{t}{\lambda}+1\right)}{\lambda \theta_{2}^{\left(\frac{t}{\lambda}+1\right)}}-\frac{\left(\frac{t}{\lambda}+1\right)^{-1} \mathrm{e}^{-\theta_{2}} \mathrm{~F}_{1}\left(1, \frac{t}{\lambda}+2, \theta_{2}\right)}{\lambda}\right] \\
& -\frac{n \alpha_{1}\left(\alpha_{2}+\alpha_{3}\right) \mathrm{e}^{\theta_{3}}}{\left[n\left(\alpha_{1}+\alpha_{3}\right)-\alpha_{3}\right]}\left[\frac{\Gamma\left(\frac{t}{\lambda}+1\right)}{\lambda \theta_{3}^{\left(\frac{t}{\lambda}+1\right)}}-\frac{\left(\frac{t}{\lambda}+1\right)^{-1} \mathrm{e}^{-\theta_{3}} \mathrm{~F}_{1}\left(1, \frac{t}{\lambda}+2, \theta_{3}\right)}{\lambda}\right] \\
& +n \alpha_{3} \mathrm{e}^{\theta_{4}}\left[\frac{\Gamma\left(\frac{t}{\lambda}+1\right)}{\lambda \theta_{4}^{\left(\frac{t}{\lambda}+1\right)}}-\frac{\left(\frac{t}{\lambda}+1\right)^{-1} \mathrm{e}^{-\theta_{4}}{ }_{1} \mathrm{~F}_{1}\left(1, \frac{t}{\lambda}+2, \theta_{4}\right)}{\lambda}\right]
\end{aligned}
$$

Now, mgf of concomitant of $r^{\text {th }}$ order statistics $Y_{[r: n]}$ can be obtained by utilizing the relation by Balasubramanian and Beg (1998):

$$
\mathrm{M}_{Y_{[r n]}}(t)=\sum_{i=n-r+1}^{n}(-1)^{i-n+r-1}\left(\begin{array}{c}
i-1 \\
n-r
\end{array}\right)\left(\begin{array}{l}
n \\
i
\end{array}\right) \mathbf{M}_{Y_{[i]]}}(t) .
$$

In view of (14), the mgf of concomitant of $r^{\text {th }}$ order statistics is given as 


$$
\begin{aligned}
& \mathrm{M}_{Y_{[r n]}}(t)= \\
& \sum_{i=n-r+1}^{n}(-1)^{i-n+r-1}\left(\begin{array}{c}
i-1 \\
n-r
\end{array}\right)\left(\begin{array}{c}
n \\
i
\end{array}\right)\left[\alpha _ { 2 } \mathrm { e } ^ { \theta _ { 1 } } \left\{\frac{\Gamma\left(\frac{t}{\lambda}+1\right)}{\left.\lambda \theta_{1}^{\left(\frac{t}{\lambda}+1\right)}-\frac{\left(\frac{t}{\lambda}+1\right)^{-1} \mathrm{e}^{-\theta_{1}} \mathrm{~F}_{1}\left(1, \frac{t}{\lambda}+2, \theta_{1}\right)}{\lambda}\right\}}\right.\right. \\
&+\frac{i \alpha_{1}\left(\alpha_{2}+\alpha_{3}\right) \mathrm{e}^{\theta_{2}}}{\left[i\left(\alpha_{1}+\alpha_{3}\right)-\alpha_{3}\right]}\left\{\frac{\Gamma\left(\frac{t}{\lambda}+1\right)}{\left.\lambda \theta_{2}^{\left(\frac{t}{\lambda}+1\right)}-\frac{\left(\frac{t}{\lambda}+1\right)^{-1} \mathrm{e}^{-\theta_{2}}{ }_{1} \mathrm{~F}_{1}\left(1, \frac{t}{\lambda}+2, \theta_{2}\right)}{\lambda}\right\}}\right. \\
&-\frac{i \alpha_{1}\left(\alpha_{2}+\alpha_{3}\right) \mathrm{e}^{\theta_{3}}}{\left[i\left(\alpha_{1}+\alpha_{3}\right)-\alpha_{3}\right]}\left\{\frac{\Gamma\left(\frac{t}{\lambda}+1\right)}{\left.\lambda \theta_{3}^{\left(\frac{t}{\lambda}+1\right)}-\frac{\left(\frac{t}{\lambda}+1\right)^{-1} \mathrm{e}^{-\theta_{3}}{ }_{1} \mathrm{~F}_{1}\left(1, \frac{t}{\lambda}+2, \theta_{3}\right)}{\lambda}\right\}}\right. \\
&\left.+i \alpha_{3} \mathrm{e}^{\theta_{4}}\left\{\frac{\Gamma\left(\frac{t}{\lambda}+1\right)}{\lambda \theta_{4}^{\left(\frac{t}{\lambda}+1\right)}}-\frac{\left(\frac{t}{\lambda}+1\right)^{-1} \mathrm{e}^{-\theta_{4}}{ }_{1} \mathrm{~F}_{1}\left(1, \frac{t}{\lambda}+2, \theta_{4}\right)}{\lambda}\right\}\right]
\end{aligned}
$$

\section{Mean of Concomitant of Order Statistics}

The $p^{\text {th }}$ moment about origin of concomitant of first order statistics $Y_{[1: n]}$ can be obtained as

$$
\mu_{Y_{[n n]]}^{p}}^{p}=\left[\frac{d^{p}}{d t^{p}}\left\{\mathrm{M}_{Y_{[n]}}(t)\right\}\right]_{t=0}
$$

Therefore, the first moment about origin i.e. mean for concomitant of first order statistics can be calculated as

$$
\mu_{Y_{[n t]]}}=\left[\frac{d}{d t}\left\{\mathrm{M}_{Y_{[[n]}}(t)\right\}\right]_{t=0}
$$

Using (14), we have 


\section{CONCOMITANT OF ORDER STATISTICS}

$$
\begin{aligned}
\mu_{\left.Y_{[n]}\right]}= & {\left[\frac { d } { d t } \left\{\alpha_{2} \mathrm{e}^{\theta_{1}}\left(\frac{\Gamma\left(\frac{t}{\lambda}+1\right)}{\lambda \theta_{1}^{\left(\frac{t}{\lambda}+1\right)}}-\frac{\left(\frac{t}{\lambda}+1\right)^{-1} \mathrm{e}^{-\theta_{1}} \mathrm{~F}_{1}\left(1, \frac{t}{\lambda}+2, \theta_{1}\right)}{\lambda}\right)\right.\right.} \\
& +\frac{n \alpha_{1}\left(\alpha_{2}+\alpha_{3}\right) \mathrm{e}^{\theta_{2}}}{\left[n\left(\alpha_{1}+\alpha_{3}\right)-\alpha_{3}\right]}\left(\frac{\Gamma\left(\frac{t}{\lambda}+1\right)}{\left.\lambda \theta_{2}^{\left(\frac{t}{\lambda}+1\right)}-\frac{\left(\frac{t}{\lambda}+1\right)^{-1} \mathrm{e}^{-\theta_{2}}{ }_{1} \mathrm{~F}_{1}\left(1, \frac{t}{\lambda}+2, \theta_{2}\right)}{\lambda}\right)}\right) \\
& \left.\left.-\frac{n \alpha_{1}\left(\alpha_{2}+\alpha_{3}\right) \mathrm{e}^{\theta_{3}}}{\left[n\left(\alpha_{1}+\alpha_{3}\right)-\alpha_{3}\right]}\left(\frac{\Gamma\left(\frac{t}{\lambda}+1\right)}{\left.\lambda \theta_{3}^{\left(\frac{t}{\lambda}+1\right)}-\frac{\left(\frac{t}{\lambda}+1\right)^{-1} \mathrm{e}^{-\theta_{3}}{ }_{1} \mathrm{~F}_{1}\left(1, \frac{t}{\lambda}+2, \theta_{3}\right)}{\lambda}\right)}\right)\right\}\right]_{t=0} \\
& \left.+n \alpha_{3} \mathrm{e}^{\theta_{4}}\left(\frac{\Gamma\left(\frac{t}{\lambda}+1\right)}{\lambda \theta_{4}^{\left(\frac{t}{\lambda}+1\right)}}-\frac{\left(\frac{t}{\lambda}+1\right)^{-1} \mathrm{e}^{-\theta_{4}}{ }_{1} \mathrm{~F}_{1}\left(1, \frac{t}{\lambda}+2, \theta_{4}\right)}{\lambda}\right)\right]
\end{aligned}
$$

Differentiating (15) with respect to $t$ and using the relationships given below:

$$
\Gamma x^{\prime}=\frac{d \Gamma x}{d x}=\psi(x) \Gamma x
$$

where $\psi(\mathrm{x})$ is the digamma function (Prudnikov et al., 1990, p. 442), we have

$$
\frac{d}{d \alpha}\left[{ }_{1} \mathrm{~F}_{1}(1 ; \alpha+v ; z)\right]=\frac{-z \mathrm{e}^{-z}}{(\alpha+v)^{2}} \mathrm{~F}_{2}(\alpha+v, \alpha+v ; \alpha+v+1, \alpha+v+1 ;-z),
$$

and setting $t=0$, 


\section{KUMAR ET AL}

$$
\begin{aligned}
& \mu_{Y_{[[n]]}}=\frac{\alpha_{2} \mathrm{e}^{\theta_{1}}}{\lambda}\left[\frac{\psi(1)-\log \theta_{1}}{\lambda \theta_{1}}+\frac{\theta_{1}}{4 \lambda}{ }^{2} \mathrm{~F}_{2}\left(2,2 ; 3,3 ;-\theta_{1}\right)+\frac{\mathrm{e}^{-\theta_{1}}}{\lambda}{ }_{1} \mathrm{~F}_{1}\left(1 ; 2 ;-\theta_{1}\right)\right] \\
& +\frac{n \alpha_{1}\left(\alpha_{2}+\alpha_{3}\right) \mathrm{e}^{\theta_{2}}}{\lambda\left[n\left(\alpha_{1}+\alpha_{3}\right)-\alpha_{3}\right]}\left[\frac{\psi(1)-\log \theta_{2}}{\lambda \theta_{2}}+\frac{\theta_{2}}{4 \lambda^{2}} \mathrm{~F}_{2}\left(2,2 ; 3,3 ;-\theta_{2}\right)\right. \\
& \left.+\frac{\mathrm{e}^{-\theta_{2}}}{\lambda}{ }_{1} \mathrm{~F}_{1}\left(1 ; 2 ;-\theta_{2}\right)\right] \\
& -\frac{n \alpha_{1}\left(\alpha_{2}+\alpha_{3}\right) \mathrm{e}^{\theta_{3}}}{\lambda\left[n\left(\alpha_{1}+\alpha_{3}\right)-\alpha_{3}\right]}\left[\frac{\psi(1)-\log \theta_{3}}{\lambda \theta_{3}}+\frac{\theta_{3}}{4 \lambda^{2}} \mathrm{~F}_{2}\left(2,2 ; 3,3 ;-\theta_{3}\right)\right. \\
& \left.+\frac{\mathrm{e}^{-\theta_{3}}}{\lambda} \mathrm{F}_{1}\left(1 ; 2 ;-\theta_{3}\right)\right] \\
& +\frac{n \alpha_{3} \mathrm{e}^{\theta_{4}}}{\lambda}\left[\frac{\psi(1)-\log \theta_{4}}{\lambda \theta_{4}}+\frac{\theta_{4}}{4 \lambda^{2}} \mathrm{~F}_{2}\left(2,2 ; 3,3 ;-\theta_{4}\right)+\frac{\mathrm{e}^{-\theta_{4}}}{\lambda}{ }_{1} \mathrm{~F}_{1}\left(1 ; 2 ;-\theta_{4}\right)\right]
\end{aligned}
$$

Now, the expression for $p^{\text {th }}$ moment about origin of concomitant of $r^{\text {th }}$ order statistics $Y_{[r: n]}$ can easily be obtained by using the relation (Siddiqui et al., 2011)

$$
\mu_{Y_{[r n]}^{p}}^{p}=\sum_{i=n-r+1}^{n}(-1)^{i-n+r-1}\left(\begin{array}{c}
i-1 \\
n-r
\end{array}\right)\left(\begin{array}{l}
n \\
i
\end{array}\right) \mu_{[[i]]}^{p} .
$$

In view of (16), the mean for concomitant of $r^{\text {th }}$ order statistics $Y_{[r: n]}$ can be obtained as 


\section{CONCOMITANT OF ORDER STATISTICS}

$$
\begin{aligned}
& \mu_{Y_{[r n]}}=\sum_{i=n-r+1}^{n}(-1)^{i-n+r-1}\left(\begin{array}{c}
i-1 \\
n-r
\end{array}\right)\left(\begin{array}{l}
n \\
i
\end{array}\right)\left[\frac { \alpha _ { 2 } \mathrm { e } ^ { \theta _ { 1 } } } { \lambda } \left\{\frac{\psi(1)-\log \theta_{1}}{\lambda \theta_{1}}\right.\right. \\
& \left.+\frac{\theta_{1}}{4 \lambda}{ }^{2} \mathrm{~F}_{2}\left(2,2 ; 3,3 ;-\theta_{1}\right)+\frac{\mathrm{e}^{-\theta_{1}}}{\lambda}{ }_{1} \mathrm{~F}_{1}\left(1 ; 2 ;-\theta_{1}\right)\right\} \\
& +\frac{i \alpha_{1}\left(\alpha_{2}+\alpha_{3}\right) \mathrm{e}^{\theta_{2}}}{\lambda\left[i\left(\alpha_{1}+\alpha_{3}\right)-\alpha_{3}\right]}\left\{\frac{\psi(1)-\log \theta_{2}}{\lambda \theta_{2}}+\frac{\theta_{2} \mathrm{e}^{-\theta_{2}}}{4 \lambda}{ }_{2} \mathrm{~F}_{2}\left(2,2 ; 3,3 ;-\theta_{2}\right)\right. \\
& \left.+\frac{\mathrm{e}^{-\theta_{2}}}{\lambda}{ }_{1} \mathrm{~F}_{1}\left(1 ; 2 ;-\theta_{2}\right)\right\} \\
& -\frac{i \alpha_{1}\left(\alpha_{2}+\alpha_{3}\right) \mathrm{e}^{\theta_{3}}}{\lambda\left[i\left(\alpha_{1}+\alpha_{3}\right)-\alpha_{3}\right]}\left\{\frac{\psi(1)-\log \theta_{3}}{\lambda \theta_{3}}+\frac{\theta_{3}}{4 \lambda^{2}} \mathrm{~F}_{2}\left(2,2 ; 3,3 ;-\theta_{3}\right)\right. \\
& \left.+\frac{\mathrm{e}^{-\theta_{3}}}{\lambda}{ }_{1} \mathrm{~F}_{1}\left(1 ; 2 ;-\theta_{3}\right)\right\} \\
& \left.+\frac{i \alpha_{3} \mathrm{e}^{\theta_{4}}}{\lambda}\left\{\frac{\psi(1)-\log \theta_{4}}{\lambda \theta_{4}}+\frac{\theta_{4}}{4 \lambda}{ }^{2} \mathrm{~F}_{2}\left(2,2 ; 3,3 ;-\theta_{4}\right)+\frac{\mathrm{e}^{-\theta_{4}}}{\lambda}{ }_{1} \mathrm{~F}_{1}\left(1 ; 2 ;-\theta_{4}\right)\right\}\right]
\end{aligned}
$$

Similarly, differentiating mgf recursively with respect to $t$, the higher order moments may be obtained.

\section{Joint Density of Concomitants of Two Order Statistics}

If $Y_{[r: n]}$ and $Y_{[s: n]}$ are the concomitant of $r^{\text {th }}$ and $s^{\text {th }}$ order statistics, respectively, then the joint pdf of $Y_{[r: n]}$ and $Y_{[s: n]}$ is given by

$$
\mathrm{g}_{[r, s: n]}\left(y_{1}, y_{2}\right)=\int_{-\infty}^{\infty} \int_{-\infty}^{x_{2}} \mathrm{f}\left(y_{1} \mid x_{1}\right) \mathrm{f}\left(y_{2} \mid x_{2}\right) \mathrm{f}_{r, s: n}\left(x_{1}, x_{2}\right) d x_{1} d x_{2},
$$

where $\mathrm{f}_{r, s: n}\left(x_{1}, x_{2}\right)$ is the joint pdf of two order statistics $X_{r: n}$ and $X_{s: n}$ and $\mathrm{f}\left(y_{1} \mid x_{1}\right)$, $\mathrm{f}\left(y_{2} \mid x_{2}\right)$ are the conditional pdf defined in (7).

If the bivariate random variables $(X, Y)$ follow $\operatorname{NBG}\left(\alpha_{1}, \alpha_{2}, \alpha_{3}, \lambda\right)$, then the joint density of concomitants of two order statistics $Y_{[r: n]}$ and $Y_{[s: n]}$ can be obtained by adding all joint densities existing at various mutually exclusive conditions, i.e., 


\section{KUMAR ET AL}

$$
\begin{aligned}
\mathrm{g}_{[r, s: n]}\left(y_{1}, y_{2}\right)=I_{1}\left(y_{1}, y_{2}\right)+I_{2}\left(y_{1}, y_{2}\right) & +I_{3}\left(y_{1}, y_{2}\right)+I_{4}\left(y_{1}, y_{2}\right) \\
& +I_{5}\left(y_{1}, y_{2}\right)+I_{6}\left(y_{1}, y_{2}\right)+I_{7}\left(y_{1}, y_{2}\right)
\end{aligned}
$$

where $I_{1}\left(y_{1}, y_{2}\right), \ldots, I_{7}\left(y_{1}, y_{2}\right)$ are the joint densities under different mutuallyexclusive conditions are and defined below.

\section{Case I: when $Y<X$}

In this case, there are the following three sub-cases:

Sub-Case 1: If $0 \leq y_{1}<x_{1}<y_{2}<x_{2} \leq \infty$.

$$
I_{1}=\int_{y_{2}}^{\infty} \int_{y_{1}}^{y_{2}} \mathrm{f}_{1}\left(y_{1} \mid x_{1}\right) \mathrm{f}_{2}\left(y_{2} \mid x_{2}\right) \mathrm{f}_{r, s: n}\left(x_{1}, x_{2}\right) d x_{1} d x_{2} .
$$

After some algebraic simplification, and in view of (7) and (10), we have

$$
\begin{array}{r}
I_{1}=\alpha_{2}^{2} \mathrm{e}^{\frac{2 \alpha_{2}}{\lambda}} \mathrm{e}^{\lambda\left(y_{1}+y_{2}\right)} \mathrm{e}^{-\frac{\alpha_{2}}{\lambda}\left(\mathrm{e}^{\lambda y_{1}}+\mathrm{e}^{\lambda y_{2}}\right)}\left(\alpha_{1}+\alpha_{3}\right)^{2} C_{r, s: n} \sum_{l=0}^{r-1} \sum_{k=0}^{s-r-1}(-1)^{l+k}\left(\begin{array}{c}
r-1 \\
l
\end{array}\right)\left(\begin{array}{c}
s-r-1 \\
k
\end{array}\right) \\
\times \mathrm{e}^{\frac{\left(\alpha_{1}+\alpha_{3}\right)(l+n-r+1)}{\lambda}} \int_{y_{2}}^{\infty} \int_{y_{1}}^{y_{2}} \mathrm{e}^{\lambda x_{1}} \mathrm{e}^{-\frac{\left(\alpha_{1}+\alpha_{3}\right)(l+s-r-k)}{\lambda} \mathrm{e}^{\lambda x_{1}}} \mathrm{e}^{\lambda x_{2}} \mathrm{e}^{-\frac{\left(\alpha_{1}+\alpha_{3}\right)(n-s+k+1)}{\lambda} \mathrm{e}^{\lambda x_{2}}} d x_{1} d x_{2}
\end{array}
$$

Solving the above equation,

$$
\begin{aligned}
I_{1}=\lambda^{4} \alpha_{2}^{2} \mathrm{e}^{\frac{2 \alpha_{2}}{\lambda}} \mathrm{e}^{\lambda\left(y_{1}+y_{2}\right)} \mathrm{e}^{-\frac{\alpha_{2}}{\lambda}\left(\mathrm{e}^{\lambda y_{1}}+\mathrm{e}^{\lambda y_{2}}\right)} C_{r, s: n} \sum_{l=0}^{r-1} \sum_{k=0}^{s-r-1}(-1)^{l+k}\left(\begin{array}{c}
r-1 \\
l
\end{array}\right)\left(\begin{array}{c}
s-r-1 \\
k
\end{array}\right) \\
\times \mathrm{e}^{\frac{\left(\alpha_{1}+\alpha_{3}\right)(l+n-r+1)}{\lambda}} \frac{\mathrm{e}^{-\frac{\left(\alpha_{1}+\alpha_{3}\right)(n-s+k+1)}{\lambda} \mathrm{e}^{\lambda x_{1}}}}{(l+s-r-k)}\left\{\mathrm{e}^{\frac{\left(\alpha_{1}+\alpha_{3}\right)(l+s-r-k)}{\lambda} \mathrm{e}^{\lambda x_{1}}}-\mathrm{e}^{-\frac{\left(\alpha_{1}+\alpha_{3}\right)(l+s-r-k)}{\lambda} \mathrm{e}^{\lambda x_{2}}}\right\}
\end{aligned}
$$

Sub-Case 2: If $0 \leq y_{1}<y_{2}<x_{1}<x_{2} \leq \infty$.

$$
I_{2}=\int_{y_{2}}^{\infty} \int_{y_{2}}^{x_{2}} \mathrm{f}_{1}\left(y_{1} \mid x_{1}\right) \mathrm{f}_{2}\left(y_{2} \mid x_{2}\right) \mathrm{f}_{r, s: n}\left(x_{1}, x_{2}\right) d x_{1} d x_{2}
$$




\section{CONCOMITANT OF ORDER STATISTICS}

Again, after some algebraic simplification and in view of (7) and (10),

$$
\begin{aligned}
I_{2}=\lambda^{3} \alpha_{2}^{2} \mathrm{e}^{\frac{2 \alpha_{2}}{\lambda}} \mathrm{e}^{\lambda\left(y_{1}+y_{2}\right)} \mathrm{e}^{-\frac{\alpha_{2}}{\lambda}\left(\mathrm{e}^{\lambda y_{1}}+\mathrm{e}^{\lambda y_{2}}\right)} C_{r, s: n} \\
\quad \times \sum_{l=0}^{r-1} \sum_{k=0}^{s-r-1}(-1)^{l+k}\left(\begin{array}{c}
r-1 \\
l
\end{array}\right)\left(\begin{array}{c}
s-r-1 \\
k
\end{array}\right) \frac{\mathrm{e}^{\frac{\left(\alpha_{1}+\alpha_{3}\right)(l+n-r+1)}{\lambda}\left(1-\mathrm{e}^{\lambda y_{2}}\right)}}{(l+n-r+1)(n-s+k+1)}
\end{aligned}
$$

Sub-Case 3: If $0 \leq y_{2}<y_{1}<x_{1}<x_{2} \leq \infty$.

$$
I_{3}=\int_{y_{1}}^{\infty} \int_{y_{1}}^{x_{2}} \mathrm{f}_{1}\left(y_{1} \mid x_{1}\right) \mathrm{f}_{2}\left(y_{2} \mid x_{2}\right) \mathrm{f}_{r, s: n}\left(x_{1}, x_{2}\right) d x_{1} d x_{2}
$$

Again, on using (7) and (10) and solving,

$$
\begin{aligned}
I_{3}=\lambda^{4} \alpha_{2}^{2} \mathrm{e}^{\frac{2 \alpha_{2}}{\lambda}} \mathrm{e}^{\lambda\left(y_{1}+y_{2}\right)} \mathrm{e}^{-\frac{\alpha_{2}}{\lambda}\left(\mathrm{e}^{\lambda y_{1}}+\mathrm{e}^{\lambda y_{2}}\right)} C_{r, s: n} \\
\quad \times \sum_{l=0}^{r-1} \sum_{k=0}^{s-r-1}(-1)^{l+k}\left(\begin{array}{c}
r-1 \\
l
\end{array}\right)\left(\begin{array}{c}
s-r-1 \\
k
\end{array}\right) \frac{\mathrm{e}^{\frac{\left(\alpha_{1}+\alpha_{3}\right)(l+n-r+1)}{\lambda}\left(1-\mathrm{e}^{\lambda y_{1}}\right)}}{(l+n-r+1)(n-s+k+1)}
\end{aligned}
$$

\section{Case II: when $X<Y$}

Again, the three sub-cases are given as

Sub-Case 1: If $0 \leq x_{1}<y_{1}<x_{2}<y_{2} \leq \infty$.

$$
I_{4}=\int_{y_{1}}^{y_{2}} \int_{0}^{y_{1}} \mathrm{f}_{1}\left(y_{1} \mid x_{1}\right) \mathrm{f}_{2}\left(y_{2} \mid x_{2}\right) \mathrm{f}_{r, s: n}\left(x_{1}, x_{2}\right) d x_{1} d x_{2}
$$

Using (7) and (10) and solving, 


\section{KUMAR ET AL}

$$
\begin{aligned}
I_{4}=\lambda^{4} & \alpha_{1}^{2}\left(\alpha_{2}+\alpha_{3}\right)^{2} \mathrm{e}^{\alpha_{2}} \mathrm{e}^{\lambda\left(y_{1}+y_{2}\right)} \mathrm{e}^{-\frac{\left(\alpha_{2}+\alpha_{3}\right)\left(\mathrm{e}^{\lambda y_{1}}+\mathrm{e}^{\lambda y_{2}}\right)}{\lambda}} \sum_{l=0}^{r-1} \sum_{k=0}^{s-r-1}(-1)^{l+k}\left(\begin{array}{c}
r-1 \\
l
\end{array}\right)\left(\begin{array}{c}
s-r-1 \\
k
\end{array}\right) \\
& \times \frac{\mathrm{e}^{\frac{\left(\alpha_{1}+\alpha_{3}\right)(l+n-r+1)}{\lambda}}}{\left[(l+s-r-k)\left(\alpha_{1}+\alpha_{3}\right)-\alpha_{3}\right]\left[(n-s+k+1)\left(\alpha_{1}+\alpha_{3}\right)-\alpha_{3}\right]} \\
\times & \left.\times \mathrm{e}^{-\frac{\left\{(l+s-r-k)\left(\alpha_{1}+\alpha_{3}\right)-\alpha_{3}\right\}}{\lambda} \mathrm{e}^{\lambda x_{1}}}\right] \\
& \times\left[\mathrm{e}^{-\frac{\left\{\left((l+s-r-k)\left(\alpha_{1}+\alpha_{3}\right)-\alpha_{3}\right\}\right.}{\lambda}}\right]
\end{aligned}
$$

Sub-Case 2: If $0 \leq x_{1}<x_{2}<y_{1}<y_{2} \leq \infty$.

$$
I_{5}=\int_{0}^{y_{1}} \int_{0}^{x_{2}} \mathrm{f}_{1}\left(y_{1} \mid x_{1}\right) \mathrm{f}_{2}\left(y_{2} \mid x_{2}\right) \mathrm{f}_{r, s: n}\left(x_{1}, x_{2}\right) d x_{1} d x_{2}
$$

Again, on using (7) and (10) and solving,

$$
\begin{aligned}
I_{5} & =\lambda^{3} \alpha_{1}^{2}\left(\alpha_{2}+\alpha_{3}\right)^{2} \mathrm{e}^{\alpha_{2}} \mathrm{e}^{\lambda\left(y_{1}+y_{2}\right)} \mathrm{e}^{-\frac{\left(\alpha_{2}+\alpha_{3}\right)\left(\mathrm{e}^{\lambda y_{1}}+\mathrm{e}^{\lambda y_{2}}\right)}{\lambda}} \\
& \times \sum_{l=0}^{r-1} \sum_{k=0}^{s-r-1}(-1)^{l+k}\left(\begin{array}{c}
r-1 \\
l
\end{array}\right)\left(\begin{array}{c}
s-r-1 \\
k
\end{array}\right) \frac{\mathrm{e}^{\frac{\left(\alpha_{1}+\alpha_{3}\right)(l+n-r+1)}{\lambda}}}{\left[(l+s-r-k)\left(\alpha_{1}+\alpha_{3}\right)-\alpha_{3}\right]} \\
& \left.\times\left[\frac{\mathrm{e}^{-\frac{\left\{(l+s-r-k)\left(\alpha_{1}+\alpha_{3}\right)-\alpha_{3}\right\}}{\lambda}}}{\left\{(n-s+k+1)\left(\alpha_{1}+\alpha_{3}\right)-\alpha_{3}\right\}}\right\} \mathrm{e}^{-\frac{\left\{(n-s+k+1)\left(\alpha_{1}+\alpha_{3}\right)-\alpha_{3}\right\}}{\lambda}}-\mathrm{e}^{-\frac{\left\{(n-s+k+1)\left(\alpha_{1}+\alpha_{3}\right)-\alpha_{3}\right\}}{\lambda} \mathrm{e}^{\lambda y_{1}}}\right\} \\
& \left.-\frac{1}{\left\{(n+l-r+1)\left(\alpha_{1}+\alpha_{3}\right)-2 \alpha_{3}\right\}}\left\{\mathrm{e}^{-\frac{\left\{(n+l-r+1)\left(\alpha_{1}+\alpha_{3}\right)-2 \alpha_{3}\right\}}{\lambda}}-\mathrm{e}^{-\frac{\left\{(n+l-r+1)\left(\alpha_{1}+\alpha_{3}\right)-2 \alpha_{3}\right\}}{\lambda} \mathrm{e}^{\lambda y_{1}}}\right\}\right]
\end{aligned}
$$




\section{CONCOMITANT OF ORDER STATISTICS}

Sub-Case 3: If $0 \leq x_{1}<x_{2}<y_{2}<y_{1} \leq \infty$.

$$
I_{6}=\int_{y_{1}}^{y_{2}} \int_{y_{1}}^{x_{2}} \mathrm{f}_{1}\left(y_{1} \mid x_{1}\right) \mathrm{f}_{2}\left(y_{2} \mid x_{2}\right) \mathrm{f}_{r, s: n}\left(x_{1}, x_{2}\right) d x_{1} d x_{2} .
$$

Again, using (7) and (10) and solving,

$$
\begin{aligned}
I_{6} & =\lambda^{3} \alpha_{1}^{2}\left(\alpha_{2}+\alpha_{3}\right)^{2} \mathrm{e}^{\alpha_{2}} \mathrm{e}^{\lambda\left(y_{1}+y_{2}\right)} \mathrm{e}^{-\frac{\left(\alpha_{2}+\alpha_{3}\right)\left(\mathrm{e}^{\lambda y_{1}}+\mathrm{e}^{\lambda y_{2}}\right)}{\lambda}} \\
& \times \sum_{l=0}^{r-1} \sum_{k=0}^{s-r-1}(-1)^{l+k}\left(\begin{array}{c}
r-1 \\
l
\end{array}\right)\left(\begin{array}{c}
s-r-1 \\
k
\end{array}\right) \frac{\mathrm{e}^{\frac{\left(\alpha_{1}+\alpha_{3}\right)(l+n-r+1)}{\lambda}}}{\left[(l+s-r-k)\left(\alpha_{1}+\alpha_{3}\right)-\alpha_{3}\right]} \\
& \times\left[\frac{\mathrm{e}^{-\frac{\left\{(l+s-r-k)\left(\alpha_{1}+\alpha_{3}\right)-\alpha_{3}\right\}}{\lambda}}}{\left\{(n-s+k+1)\left(\alpha_{1}+\alpha_{3}\right)-\alpha_{3}\right\}}\left\{\mathrm{e}^{-\frac{\left\{(n-s+k+1)\left(\alpha_{1}+\alpha_{3}\right)-\alpha_{3}\right\}}{\lambda}}-\mathrm{e}^{-\frac{\left\{(n-s+k+1)\left(\alpha_{1}+\alpha_{3}\right)-\alpha_{3}\right\}}{\lambda} \mathrm{e}^{\lambda y_{2}}}\right\}\right. \\
& \left.-\frac{1}{\left\{(n+l-r+1)\left(\alpha_{1}+\alpha_{3}\right)-2 \alpha_{3}\right\}}\left\{\mathrm{e}^{-\frac{\left\{(n+l-r+1)\left(\alpha_{1}+\alpha_{3}\right)-2 \alpha_{3}\right\}}{\lambda}}-\mathrm{e}^{-\frac{\left\{(n+l-r+1)\left(\alpha_{1}+\alpha_{3}\right)-2 \alpha_{3}\right\}}{\lambda} \mathrm{e}^{\lambda y_{2}}}\right\}\right]
\end{aligned}
$$

\section{Case III: When $X=Y$}

$$
I_{7}=\mathrm{f}_{3}\left(y_{1} \mid x_{1}\right) \mathrm{f}_{3}\left(y_{2} \mid x_{2}\right) \mathrm{f}_{r, s: n}\left(x_{1}, x_{2}\right) .
$$

On using (7) and (10),

$$
\begin{aligned}
I_{7}= & \alpha_{3}^{2} \mathrm{e}^{\frac{2 \alpha_{3}}{\lambda}} \mathrm{e}^{\lambda\left(y_{1}+y_{2}\right)} \mathrm{e}^{-\frac{\alpha_{3}}{\lambda}\left(\mathrm{e}^{\lambda y_{1}}+\mathrm{e}^{\lambda y_{2}}\right)} C_{r, s: n} \\
& \times \sum_{l=0}^{r-1} \sum_{k=0}^{s-r-1}(-1)^{l+k}\left(\begin{array}{c}
r-1 \\
l
\end{array}\right)\left(\begin{array}{c}
s-r-1 \\
k
\end{array}\right) \mathrm{e}^{\frac{\left(\alpha_{1}+\alpha_{3}\right)(l+n-r+1)}{\lambda}} \mathrm{e}^{-\frac{\left(\alpha_{1}+\alpha_{3}\right)(l+s-r-k)}{\lambda} \mathrm{e}^{\lambda y_{1}}} \mathrm{e}^{-\frac{\left(\alpha_{1}+\alpha_{3}\right)(n-s+k+1)}{\lambda} \mathrm{e}^{\lambda y_{2}}} .
\end{aligned}
$$

Putting the values of $I_{1}, \ldots, I_{7}$ in (17), obtain the required joint density of two concomitant of order statistics. 


\section{KUMAR ET AL}

\section{References}

Abramowitz, M., \& Stegun, I. A. (1972). Handbook of mathematical functions $\left(9^{\text {th }}\right.$ edition). New York: Dover Publications, Inc.

Balasubramanian, K., \& Beg, M. I. (1996). Concomitant of order statistics in bivariate exponential distribution of Marshall and Olkin. Calcutta Statistical Association Bulletin, 46(1-2),109-115. doi: 10.1177/0008068319960109

Balasubramanian, K., \& Beg, M. I. (1997). Concomitant of order statistics in Morgenstern type bivariate exponential distribution. Journal of Applied Statistical Science, 54(4), 233-245.

Balasubramanian, K. \& Beg, M. I. (1998). Concomitant of order statistics in Gumbel's bivariate exponential distribution. Sankhyā: The Indian Journal of Statistics, Series B, 60(3), 399-406.

Begum, A. A. (2003). Concomitant of order statistics from bivariate Pareto II distribution. International Journal of Statistical Sciences, 2, 27-35.

Begum, A. A., \& Khan, A. H. (2000). Concomitant of order statistics from Marshall and Olkin's bivariate Weibull distribution. Calcutta Statistical Association Bulletin, 50(1-2), 65-70. doi: 10.1177/0008068320000106

Bhattacharya, P. K. (1984). Induced order statistics: Theory and applications. In P. R. Krishnaiah \& P. K. Sen (Eds.), Handbook of statistics 4: Nonparametric Methods (pp. 383-403). New York: Elsevier. doi: 10.1016/S01697161(84)04020-7

Chacko, M., \& Thomas, P. Y. (2011). Estimation of parameter of Morgenstern type bivariate exponential distribution using concomitant of order statistics. Statistical Methodology, 8(4), 363-376. doi:

10.1016/j.stamet.2011.02.004

David, H. A. (1973). Concomitant of order statistics. Bulletin of the International Statistical Institute, 45, 295-300.

David, H. A. (1981). Order statistics (2 ${ }^{\text {nd }}$ edition). New York: John Wiley.

El-Sherpieny, E. A., Ibrahim, S. A., \& Bedar, R. E. (2013). A new bivariate distribution with Gompertz marginal. International Journal of Basic and Applied Sciences, 2(4), 348-355.

Philip, A., \& Thomas, P. Y. (2015). On concomitants of order statistics arising from the extended Farlie-Gumble-Morgenstern bivariate logistic distribution and its application in estimation. Statistical Methodology, 25, 59-73. doi: 10.1016/j.stamet.2015.02.002 


\section{CONCOMITANT OF ORDER STATISTICS}

Prudnikov, A. P., Brychkov, IU. A., Marichev, O. I., \& Gould, G. G. (1990). Integrals and series: More special functions (Vol. 3). New York: Gordon and Breach Science Publishers.

Siddiqui, S. A., Jain, S., Siddiqui, I., Alam, M., \& Chalkoo, P. A. (2011). Moments and joint distribution of concomitant of order statistics. Journal of Reliability and Statistical Studies, 4(2), 25-32. Retrieved from http://jrss.in.net/assets/4102.pdf

Thomas, P. Y., \& Veena, T. G. (2011). On an application of concomitants of order statistics in characterizing a family of bivariate distributions.

Communications in Statistics - Theory and Methods, 40(8), 1445-1452. doi: 10.1080/03610921003606319

Yang, S. S. (1977). General distribution theory of the concomitants of order statistics. Annals of Statistics, 5(5), 996-1002. doi: 10.1214/aos/1176343954 